\title{
SYNTHETIC APERTURE RADAR INTERFEROMETRY FOR DIGITAL ELEVATION MODEL OF KUWAIT DESERT - ANALYSIS OF ERRORS
}

\author{
Hala K. Al Jassar ${ }^{\text {a }}$, and K.S. Rao ${ }^{\mathrm{b}}$ \\ ${ }^{\text {a }}$ Physics Department, Kuwait University, Kuwait - hala_62@hotmail.com \\ b Physics Department, Kuwait University, Kuwait - ks.rao@ku.edu.kw
}

KEY WORDS: Digital Elevation Model, SAR interferometry, errors, weighted average, ASAR, atmospheric corrections

\begin{abstract}
:
Using different combinations of 29 Advanced Synthetic Aperture Radar (ASAR) images, 43 Digital Elevations Models (DEM) were generated adopting SAR Interferometry (InSAR) technique. Due to sand movement in desert terrain, there is a poor phase correlation between different SAR images. Therefore, suitable methodology for generating DEMs of Kuwait desert terrain using InSAR technique were worked out. Time series analysis was adopted to derive the best DEM out of 43 DEMs. The problems related to phase de-correlation over desert terrain are discussed. Various errors associated with the DEM generation are discussed which include atmospheric effects, penetration into soil medium, sand movement. The DEM of Shuttle Radar Topography Mission (SRTM) is used as a reference. The noise levels of DEM of SRTM are presented.
\end{abstract}

\section{INTRODUCTION}

DEMs are widely known for their applications in Civil Engineering, alignment of transport, power lines, drainage systems, communication networks etc. In Kuwait, it has great importance for the alignment of oil pipe lines. There are various techniques for generating DEM such as cartographic, satellite stereo-graphic and Synthetic Aperture Radar Interferometry (InSAR). During the recent years, InSAR technique has been considerably improved to generate accurate and high vertical/spatial resolutions DEMs (Zhang et al., 2005; Goncalves et al., 2008; Rao et al., 2006; Rodriguez et al., 2006; Hofton et al., 2006) . During the past 5 years, good amount of work is done in Kuwait ( Rao and Hala (2010a,b,c), Hala and Rao (2011)) using SAR Interferometry technique for generating DEM and also land subsidence. This paper summarizes our results so far obtained and published in referred journals.

\section{STUDY AREA}

The study are is Kuwait (see Figure 1). Since SAR Interferometry has a decorrelation problem over open areas of the desert, the study area is confined to Oil fields of Kuwait. The main oil fields are Burgan, Managesh and Umgadir. Since Managesh and Umgadir oil fields are very close, in this paper, they are referred to as Managesh. The climate of Kuwait are given by Hala et al. (2006).

\section{DATASETS AND SOFTWARE PACKAGES USED IN THIS STUDY}

A variety of data sets are utilized in this study. Twenty nine ASAR images acquired from EnviSat Satellite are used. An example of ASAR image of Kuwait is shown in figure 1. The protected areas (oil fields) are clearly separated from the open desert. Twenty seven water vapour images from MERIS onboard ENVISAT are used, An example of water vapor image of Kuwait and its surrounding regions is shown in Figure 2. Topomaps of Kuwait, DEM of SRTM at $90 \mathrm{~m}$ spatial

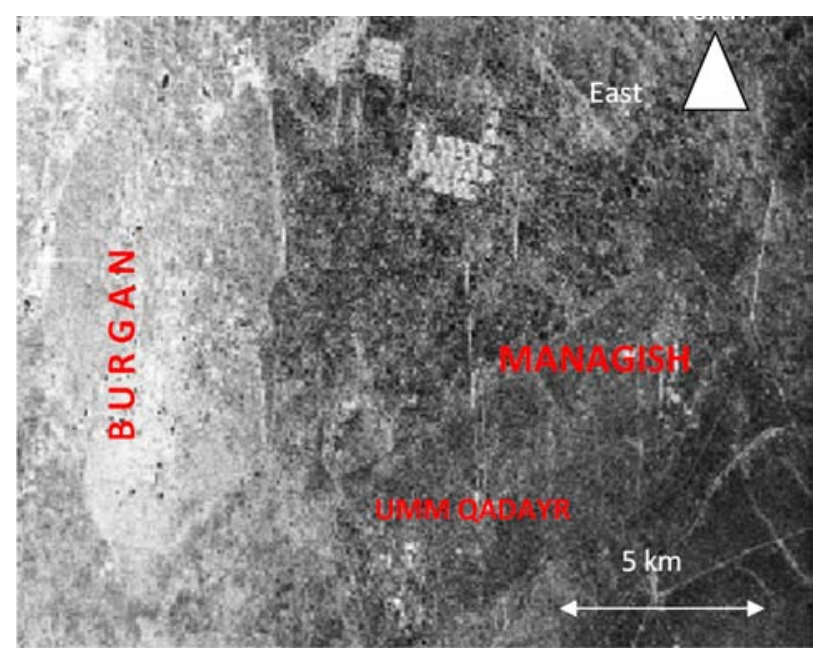

Figure 1. ASAR intensity image of a part of Kuwait with major oil fields. Since the data is for Descending pass, East and West are interchanged. The low contrast area on the right side refers to open desert sand. The bright portion on the left is Burgan oil field. This image corresponds to March 2007.

resolution, soil moisture data from AMSR-E are used in this study. The monthly average of soil moisture over Kuwait is shown in Figure 3. GAMMA interferometric software, ERDAS image processing software and EoliSA packages are used for the analysis of the data. Special software was developed by our group at Physics Department, Kuwait University for the analysis and presentation of the results.

\section{GENERATION DEMS USING INSAR TECHNIQUE}

Generating DEMs using InSAR technique is well documented and published in our earlier publications. The flowchart is shown in Figure 4. 


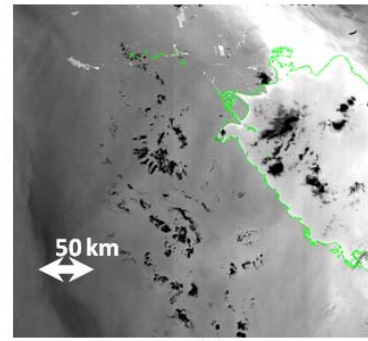

(a)

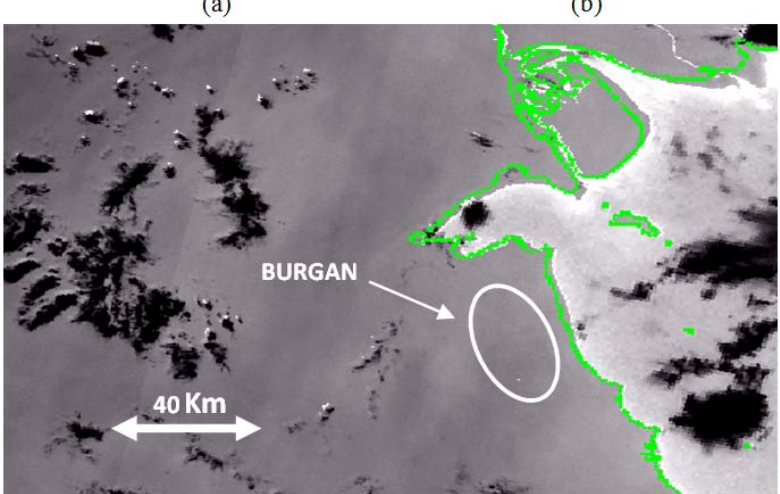

(c)

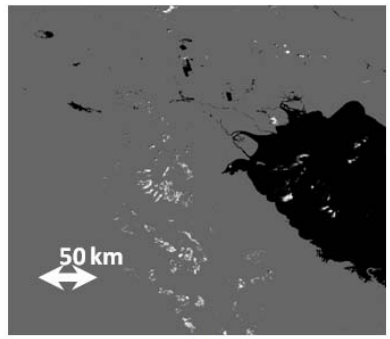

(b) (a) Full scene of MERIS PWV

Figure 2. Water vapor images. (a) Full scene of MERIS PWV. (b) Cloud albedo of MERIS PWV (c) Kuwait scene of MERIS PWV.

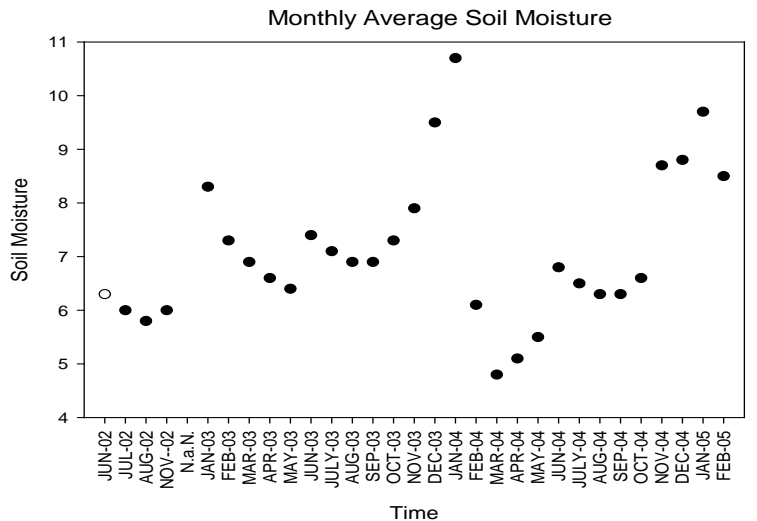

Figure 3. Monthly average soil moisture corresponding to a pixel (50 km circular diameter) of Kuwait desert.

The technique is specially tuned to suite to Kuwait desert environmental conditions. The major problem associated with the desert is the movement of sand which destroys the coherence between the pair of images required for generating Interferogram. Even a temporal base line of 35 days are enough to destroy the coherence. However, for the protected areas like oil fields, the coherence is preserved by the scant vegetation that was grown during the periods of rain fall. The multi-year scrubs acts as a barrier for the sand movement. In the open desert area, animal grazing destroys the vegetation cover, thus allowing the sand to move due to desert wind. Therefore, particularly in Kuwait desert, InSAR technique works only over oil fields, military sites and city area. Kuwait is covered by two major oil fields - Burgan and Managuish/Umgadir. The results presented here refers to these two oil fields.

Though a large number of pairs are possible with 29 ASAR scenes, only 43 pairs are considered in this study whose base line varies between 100 and $400 \mathrm{~m}$. Figure 5 shows the temporal baseline (day-difference) vrs. Spatial baseline. For higher baselines, decorrelation of phase is a problem. For lower baselines, DEM sensitivity is an issue. Shorter the temporal baseline, better correlation and so reliability of the results. In time series analysis, less weightage is given for high temporal baseline pairs.

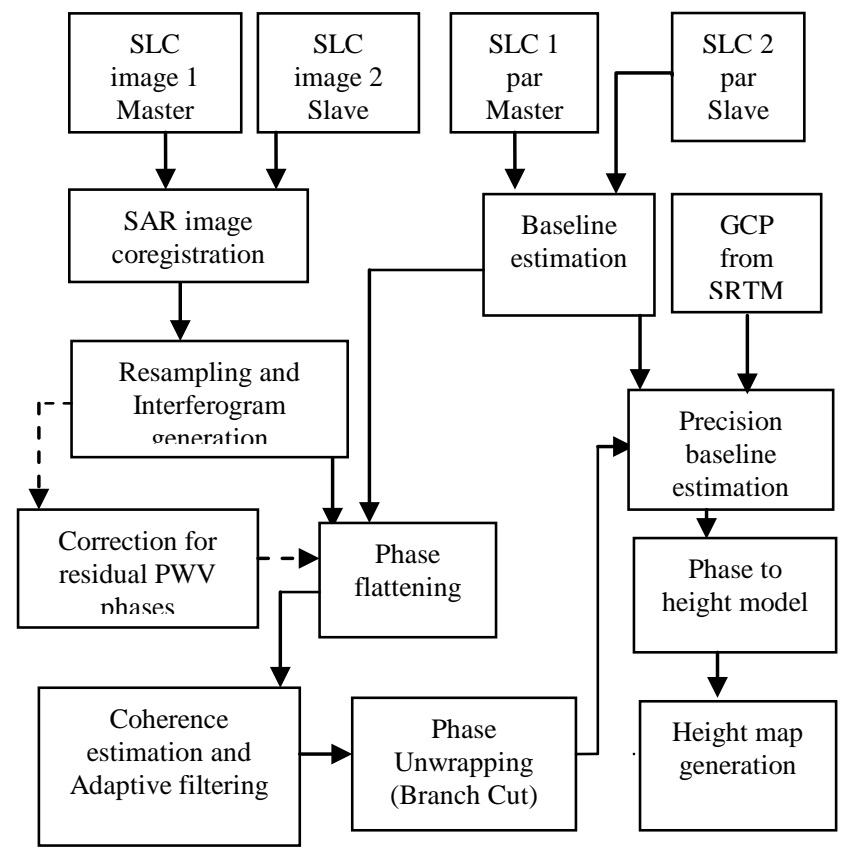

Figure 4. Flow chart for generating DEM through InSAR technique. The details of various steps are given in the text.

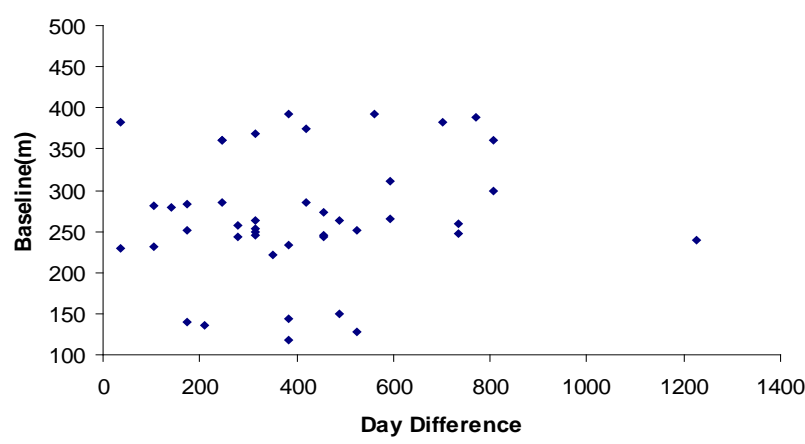

Figure 5. Baseline ( $\boldsymbol{B}_{\perp}$ ) as a function of day-difference shows the distribution of interferometric pairs used in this study. There are 43 pairs whose $\boldsymbol{B}_{\perp}$ varies between $150-400 \mathrm{~m}$. The day-difference varies between 35 - 770 except the case of one pair whose day-difference is 1225 days.

\section{TIME SERIES GENERATION OF DEM OF KUWAIT AND RESULTS}

Twenty nine ASAR scenes acquired during the time period 2005-2010 are used to generate 43 DEMs. All the generated DEMs are supposed to be same in elevations. However, there is not much correlation found among different DEMs of Kuwait oil fields. One possible reason is the effect of water vapor relative delays. Using MERIS water vapor data, corrections are incorporated which does not improve the accuracies. This is due to small quantity of residual water vapor and poor 
approximations in the model. Another reason was the temporal and spatial variation of soil moisture (see Figure 3). The more the soil moisture - less the penetration of microwaves. The uneven penetration of microwaves creates additional phases which are mistakenly interpreted as elevations. The third reason is the coherence between the pair of images used for generating Interferogram. Though the occasional correlation coefficient (CC) is as high as 0.9 , the average of the scene is not more than 0.5. Therefore, this will cause additional errors in the DEMs.

To minimize the errors, time series analysis technique is applied. First-of-all, all the DEMs are registered to one another. The elevations of a particular pixel are extracted to find the mean and standard deviation (STD) of elevations. The data is filtered by setting the condition mean \pm STD. All the elevations satisfying the above condition are collected and the new mean elevation is estimated. This process is repeated for all the pixels in the interferogram to generate the elevation image. This procedure considerably reduced the errors in the final DEM. The resultant DEM of Burgan oil field and Mamagesh oil field are shown in Figures 6,7.

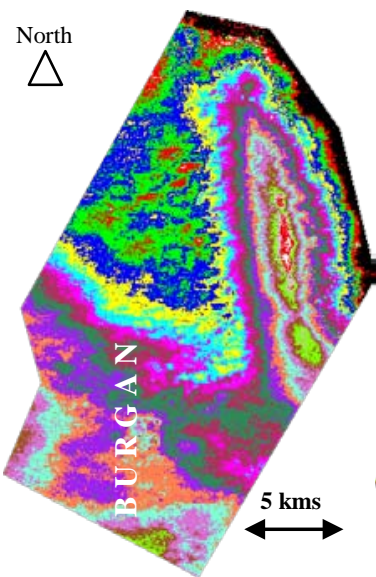

(a)

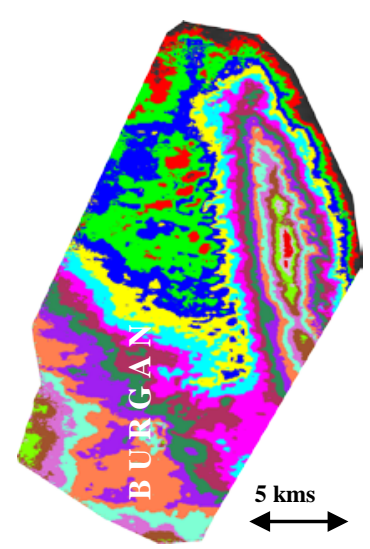

(c)

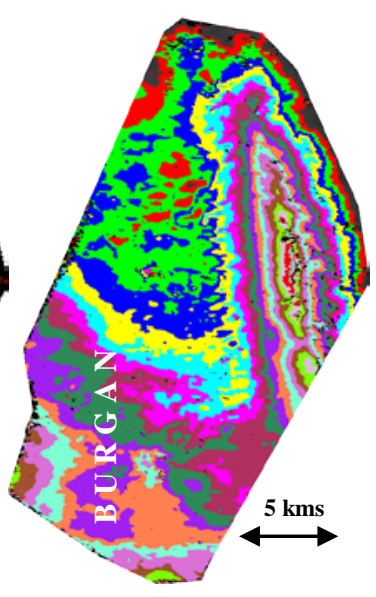

(b)

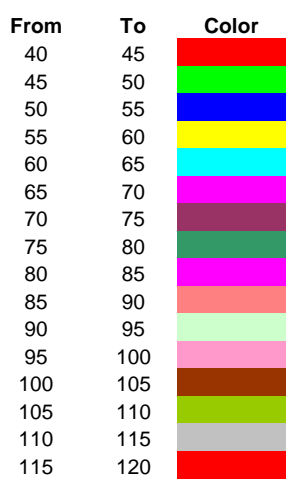

Figure 6. Colour coded and geocoded DEMs of Burgan oil field. The colour coding is at an interval of $5 \mathrm{~m}$. (a) derived from SRTM, (b) derived from the pair with least STD error ( $2.8 \mathrm{~m}$ ) corresponding to the pair December 2007 - July 2007. The day-difference in this case is 140 days and the $\boldsymbol{B}_{\perp}$ is 280 m., (c) the weighted average image taking into account all the 43 DEMs. The values are in meters in the color coding.

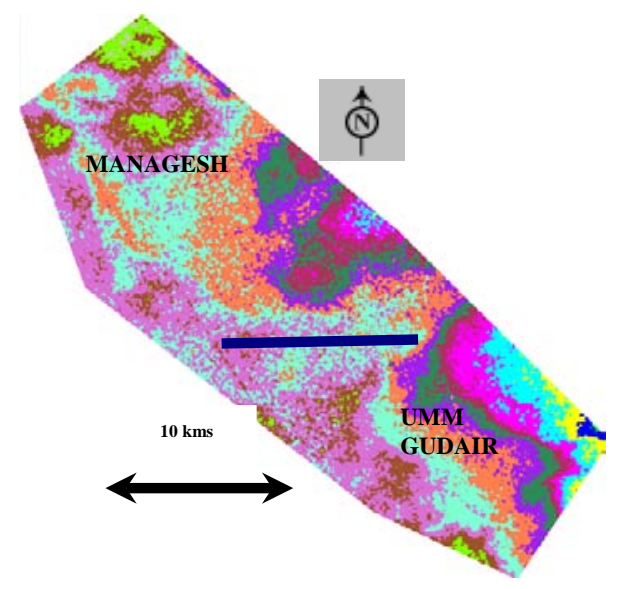

(a)

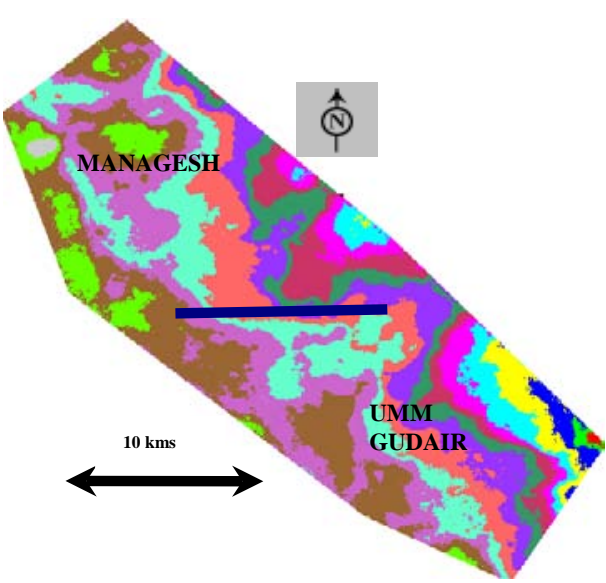

(b)

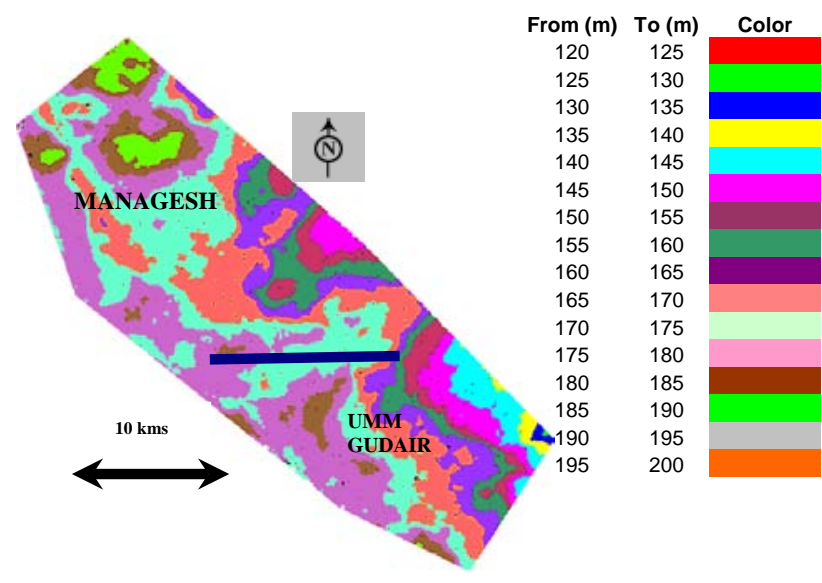

(c)

Figure 7. (a) SRTM (b) Weighted average DEM (c) InSAR Pair Dec 07 - July 07. Comparison of DEMs of SRTM, Weighted average InSAR DEM and the DEM of one pair with lesser RMS error. There is a general agreement reflecting the topography of the study area. However, SRTM DEM shows more noise and InSAR DEMs are very smooth. 


\section{DISCUSSION OF RESULTS}

Following the time series analysis approach as explained above, InSAR DEMs of Kuwait oil fields are compared with the DEM of SRTM at $90 \mathrm{~m}$ resolution as shown in Figure 6 and 7. Overall, there is a good agreement. However, DEM of SRTM shows more local undulations compared to repeat pass InSAR DEM which is difficult to explain. Since the two images are taken simultaneously in SRTM, it must be free from all the error sources. Inspite of the fact, it is showing more local undulations. A small portion of Managesh oil field data is extracted and shown in Figure 8.

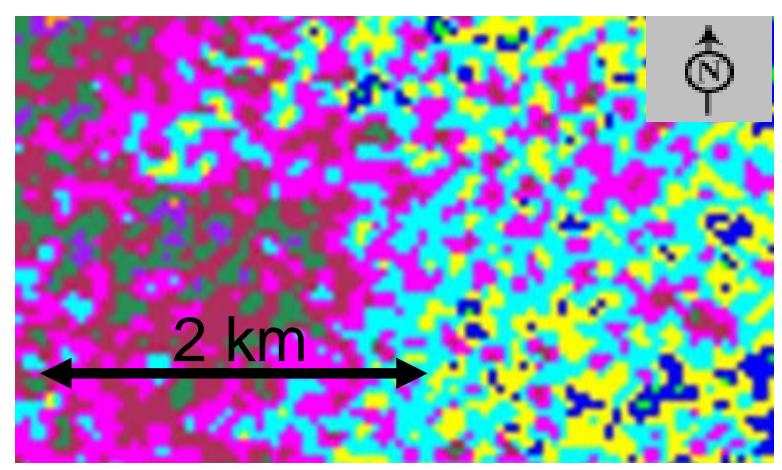

(a) SRTM DEM

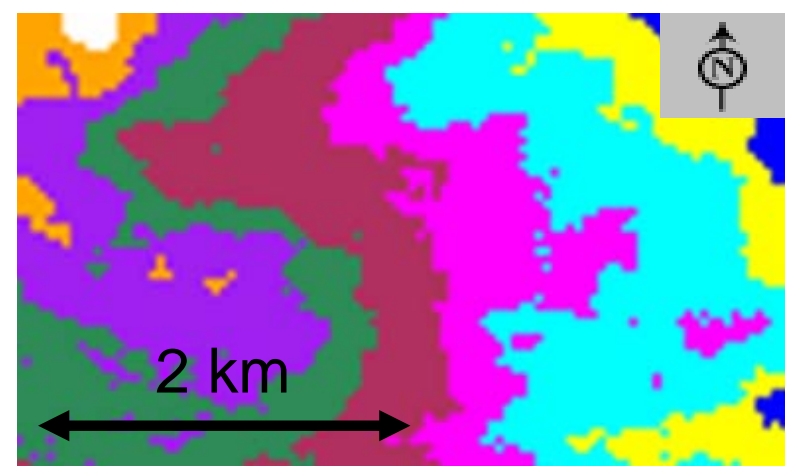

(b) Weighted Average DEM

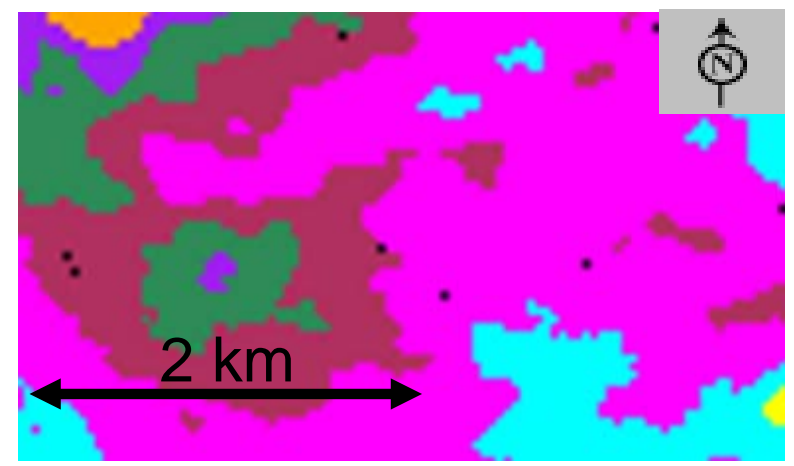

(c) InSAR Pair Dec 07 - Jul 07

Figure 8. A small portion of the DEM of Figure 8 is enlarged and color coded at $2 \mathrm{~m}$ interval to enhance the noise levels. Center Coordinates are: - 28 $52^{\prime} 30^{\prime \prime} \mathrm{N}$ and $47^{\circ} 38^{\prime} 21^{\prime \prime} \mathrm{E}$

The noise level in SRTM DEM is very clear. The possible reasons can be the possible vibrations of $80 \mathrm{~m}$ shaft to achieve required baseline for InSAR technique to work. Figure 9 shows a profile of elevations taken from the DEM as shown in Figure 8. Mean filter is used to estimate the noise in the DEM of SRTM and computed as $1 \mathrm{~m}$.

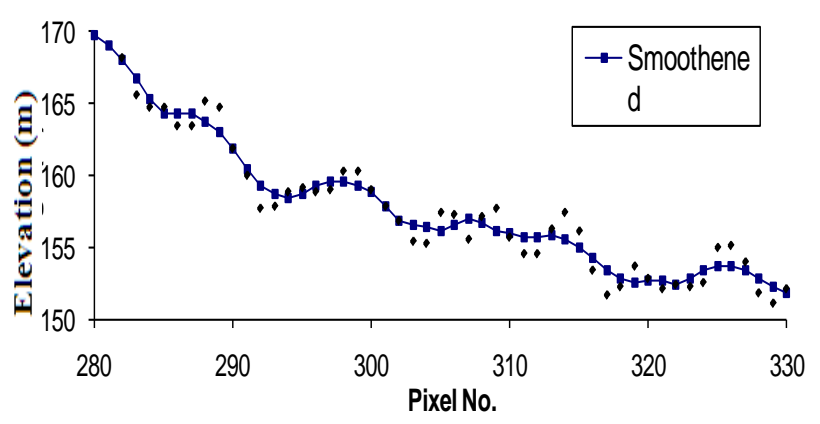

Figure 9. Illustration of noise levels of SRTM DEM and the filtered smooth curve. The Standard Deviation is computed to be $0.99 \mathrm{~m}$

\section{CONCLUSIONS}

We are successful in generating DEM of Kuwait desert terrain in-spite of low coherence due to sand movement, high penetration of waves into desert soils, spatial and temporal distribution of precipitable water vapor and many other factors. Our results correlate well with the DEM of SRTM. It is observed that the DEM of SRTM shows more local undulations and noisy. The noise level is estimated to be $1 \mathrm{~m}$ by using mean filter.

\section{ACKNOWLEDGEMENT}

The authors are thankful to KFAS for sponsoring the research under project no. 2010440103.

\section{REFERENCES}

Goncalves, J.A and Morgado A. M., 2008. Use of the SRTM DEM as a geo-referencing tool by elevation matching. Int. Arch. Photogram. Rem. Sens. Spatial Inform. Sci., 37(B2), pp. 879-883.

Hala, K. and Rao, K. S., 2011. Observation of atmospheric effects on Synthetic Aperture Radar repeat pass interferometry over Kuwait Desert. International Journal of Remote Sensing, 32(22), pp. 7613-7629.

Hala, K., Rao, K. S. and Sabbah I., 2006. A model for the retrieval and monitoring of soil moisture over desert area of Kuwait. International journal of remote sensing, 27(1-2), pp. 329-348.

Hofton M., Dubayah, R. J., Blair, J. B. and Rabine, D., 2006. Validation of SRTM elevations over vegetated and nonvegetated terrain using medium footprint LIDAR. Photogramm. Eng. Rem. Sens., 72(3), pp. 279-285. 
Rao, K. S. and Hala K., 2010a. Assessment of Shuttle Radar Topographic Mission performance over oil fields of Kuwait desert terrain. J. Appl. Remote Sens., 6(063504).

Rao, K. S. and Hala K., 2010b. Digital elevation model of Burgan oil field, Kuwait derived from repeat pass satellite radar interferometry. Kuwait Journal of Science and Engineering, 37(2A), pp. 159-174.

Rao, K. S. and Hala K., 2010ca. Error analysis in the digital elevation model of Kuwait desert derived from repeat pass synthetic aperture radar interferometry. J. Appl. Remote Sens., 4(043456).

Rao, K.S., Phalke, S. M., Sakalley J. and Al-Jassar, H.K., 2006. Assessment of Geo-coding and Height Accuracy of the DEM derived from preliminary data sets of X-band SRTM. Indian Soc. Rem. Sens., 34, pp. 369-375.

Rodriguez, E., Morris, C. S. and Belz, E., 2006. A global assessment of the SRTM performance. Photogramm. Eng. Rem. Sens., 72, pp. 249-260.

Zhang, Y. and Cheng, X. Application of SRTM-DEM based two-pass SAR interferometry for detecting seismic deformation on high-altitude rugged terrain - A case study in Kokoxili Ms 8.1 earthquake. IEEE Int. Geosci. Rem. Sens. Symp., 7, pp. 5316-5319. 